\title{
ELEMENTOS PARA LA CONSTRUCCIÓN DE UN INSTRUMENTO QUE PERMITA MEDIR LA INTENCIÓN EMPRESARIAL EN ESTUDIANTES UNIVERSITARIOS DE ÚLTIMO SEMESTRE ${ }^{1}$
}

\author{
Por: Edwin Tarapuez Chamorro ${ }^{2}$, María Dolly García González ${ }^{3}$, Nélida Castellano ${ }^{4}$
}

\section{RESUMEN}

El objetivo de este artículo es construir un instrumento que permita identificar factores que influyen en la intención de crear empresas, en los estudiantes universitarios de último semestre, de carreras profesionales presenciales del departamento del Quindío (Colombia), utilizando la Teoría del Comportamiento Planeado de Ajzen. La metodología utilizada consiste en diseñar una encuesta inicial con base en la revisión de la literatura existente, que posteriormente se valida mediante las opiniones de 14 expertos y se priorizan las preguntas por parte de 51 "estudiantes jueces", para seleccionar, por el método de los cuartiles, aquellas que discriminan. El resultado es la encuesta depurada que incluye ítems sobre aspectos personales y familiares, Normas sociales, Imagen y valores, Creencias, aspectos que motivan y obstaculizan la creación de empresas, y preguntas sobre recursos. Los resultados de la aplicación de esta encuesta, hacen parte del proyecto de investigación: "Factores que influyen en la intención de creación de empresas en los estudiantes universitarios del departamento del Quindío (Colombia)".

Palabras clave: priorización de preguntas, juicio de expertos, intención empresarial, creación de empresas, emprendimiento.

JEL: M19.

1. El presente artículo corresponde a los resultados parciales de la tesis doctoral titulada "Factores que influyen en la intención de creación de empresas en los estudiantes universitarios del departamento del Quindío (Colombia)”, que el autor principal desarrolla actualmente con la asesoría metodológica de la Profesora García y bajo la dirección de la Dra. Castellano.

2. Doctorando en Ciencias Económicas (Universidad Nacional de Córdoba, Argentina). Docente asociado de la Universidad del Quindío. Email: eitarapuez@uniquindio.edu.co.

3. Magíster en Matemáticas (Universidad Nacional de Colombia). Docente titular de la Universidad del Quindío. Email: mdgarcia@uniquindio.edu.co

4. Doctora en Ciencias Económicas (Universidad Nacional de Córdoba, Argentina). Profesora Titular por Concurso de la Universidad Nacional de Córdoba (Argentina). Email: ncastell@eco.unc.edu.ar

Artículo recibido: 26 de marzo de 2015.

Aprobación definitiva: 30 de mayo de 2015 


\title{
ELEMENTS FOR BUILDING AN INSTRUMENT TO MEASURE ENTREPRENEURSHIP IN LAST TERM BACHELOR STUDENTS
}

\author{
By: Edwin Tarapuez Chamorro, María Dolly García González, Nélida Castellano
}

\begin{abstract}
The main goal of this paper is to build an instrument to identify factors influencing entrepreneurship in Quindío department's (Colombia) last term, face to face bachelor students, using the Theory of Planned Behavior of Ajzen. An initial survey is designed based on literature review, the survey is later validated by 14 experts' opinions and questions are prioritized by 51 "student judges", to select, by using the quartiles method, those that they discriminate. The result is a refined survey that includes family and personal issues ítemes, questions in the following dimensions: Social rules, Image and Values, Beliefs, Aspects that motivate business creation and Obstacles, and questions about resources. The results of conducting this survey, are part of the "Factors that influence entrepreneurship in university students of Quindío department (Colombia) research project.
\end{abstract}

Keywords: question prioritizing, expert opinion, entrepreneurship, business creation.

JEL: M19 


\title{
ELEMENTOS PARA A CONSTRUÇÃO DE UM INSTRUMENTO QUE PERMITA MEDIR A INTENÇÃO EMPRESARIAL EM ESTUDANTES UNIVERSITÁRIOS DE ÚLTIMO SEMESTRE
}

\author{
Por: Edwin Tarapuez Chamorro, María Dolly García González, Nélida Castellano
}

\section{RESUMO}

O principal objetivo deste artigo é construir um instrumento que permita identificar fatores que influenciem na intenção de criar empresas nos estudantes universitários de último semestre de programas profissionalizantes e de modalidade presencial no departamento do Quindío (Colômbia), usando a Teoria da Planejado Comportamento de Ajzen. A metodologia utilizada consiste em desenhar um questionário inicial, com base na revisão da literatura existente, que posteriormente é validado através das opiniões de 14 expertos y se priorizam as questões por parte de 51 "estudantes juízes" para selecionar, pelo método dos quantis, aquelas que discriminam. $O$ resultado é um questionário que inclui aspectos pessoais e familiares, questões nas dimensões: Normas sociais, Imagem e valores, Crenças, Aspectos que motivam a criação de empresas e obstáculos para criar empresas, além de questões sobre recursos. Os resultados da aplicação do questionário, fazem parte do projeto de pesquisa: "Fatores que influenciam na intenção de criação de empresas nos estudantes universitários do departamento do Quindío, (Colombia)".

Palavras-chave: priorização de perguntas, avaliação de expertos, intenção empresarial, criação de empresas, empreendedorismo.

JEL: M19 


\section{INTRODUCCIÓN}

La intencionalidad puede ser definida como un estado de la mente que dirige la atención de las personas, la experiencia y la acción hacia un objetivo específico o un camino para alcanzar algo (Bird, 1988); por lo tanto, la acción empresarial puede ser clasificada también como un comportamiento intencional que forma parte de la cadena de valor de la creación de empresas (Krueger, 1993; Baum, et al,. 2007). En este sentido, para Gatewood, et al. (1995), la intención de crear empresa está unida a las actitudes, más específicamente a la deseabilidad percibida y la factibilidad, en tanto que para Gasse \& D’Amours (2000), las características consideradas propias de los creadores de empresa sólo influyen en las intenciones de montar una unidad productiva cuando afectan las creencias y las percepciones de los individuos.

Existen muchas teorías y modelos que buscan explicar el proceso que siguen los individuos para crear una empresa; una de estas teorías es la de la conducta prevista (Ajzen, 1991, 1988, 1987, 1985), que provee un marco general para analizar la intención individual de crear una empresa. Esta teoría, propuesta a principios de los años 80, ha sido utilizada en el análisis de problemas de medicina, drogadicción, tabaquismo, relaciones públicas, campañas de publicidad, medio ambiente y uso del tiempo libre, entre otras, y ha demostrado ser muy útil a la hora de abordar el estudio de la intención empresarial.

Shapero (1984), uno de los primeros autores en utilizar la teoría de la conducta prevista en el área empresarial, indicó que la intención empresarial se deriva de una percepción de viabilidad y deseabilidad de una persona y este camino es afectado por el contexto cultural y social.

Con base en los dos modelos mencionados, se ha propuesto un enfoque centrado en procesos, que ha sido ampliamente utilizado por un grupo significativo de académicos (Krueger, 1993; Krueger \& Brazeal, 1994; Krueger \& Carsrud, 1993), sin embargo, son pocas las investigaciones que han intentado comprender la forma cómo los valores, las actitudes y los comportamientos pueden hacer que las personas tengan predisposición para crear empresa o para que tengan la intención de hacerlo (Gasse \& Tremblay, 2011).

En general, muchos estudios anteriores han encontrado que la intención emprendedora está determinada por un conjunto variado de factores (Hisrich, et al., 2004; Kuratko \& Hodgetts, 2006; Liñán, 2008; Nabi \& Holden, 2008; Harris \& Gibson, 2008; Jones, et al., 2008). Las investigaciones se enfocan en dos líneas: una con relación a los factores personales que hacen a los emprendedores diferentes al resto de la población y otra vinculada con los factores ambientales, tales como las características socioeconómicas y demográficas.

Uno de los elementos centrales a considerar es la intención individual de crear una empresa y poner en práctica un comportamiento específico influido por elementos motivacionales, sin embargo, la teoría de la conducta prevista no incorpora en sus análisis, características de la personalidad, variables socioeconómicas, factores de índole social, ni aspectos que obstaculicen la creación de empresas, que necesariamente están relacionados con la conducta de los individuos. 


\section{MATERIALES Y MÉTODOS}

Dado que en la revisión de literatura no se contó con un instrumento que tuviera en cuenta el contexto colombiano o latinoamericano se procedió, en primer lugar, a diseñar un cuestionario estructurado a partir de diversos autores que han trabajado el tema de intención empresarial; este cuestionario luego se validó a través de la discusión con 14 expertos en el tema de creación de empresas y aspectos académico-investigativos relacionados; posteriormente se priorizaron los ítemes más discriminantes con otro grupo de 51 "estudiantes jueces"; después se realizó una prueba piloto con un grupo de 30 estudiantes y finalmente se discutió el modelo definitivo de encuesta con un subgrupo de los 14 expertos iniciales.

\section{DISEÑO DE LA ENCUESTA}

\section{a) Definición de dimensiones y atributos}

Se partió del marco conceptual proporcionado por la Teoría del Comportamiento Planeado (Ajzen, 1991, 1988, 1987, 1985), que identifica actitudes hacia el comportamiento, normas subjetivas y control de comportamiento percibido, los cuales en conjunto determinan la intención de crear empresa y posteriormente pueden concretarse en la creación de nuevas unidades productivas; para identificar los atributos correspondientes se realizó la revisión bibliográfica del caso, la cual se presenta en la Tabla 1, donde se reúnen las dimensiones, variables y autores de quienes se tomó información para adaptar las preguntas de la encuesta inicial.

\section{b) Selección de preguntas y confección del modelo de encuesta}

Con la información referida en la Tabla 1 se realizó una selección inicial de 50 preguntas para ser valoradas en una escala tipo Likert, relacionadas solamente con las dimensiones: Normas sociales, Imagen y valores del empresario quindiano, Creencias, Motivaciones (actitudes positivas) y Obstáculos para crear empresa (actitudes negativas).

\section{VALIDACIÓN}

\section{a) Validez del contenido}

Para evaluar la validez del cuestionario de 50 ítemes, se consideraron inicialmente las opiniones de 14 líderes, vinculados con el desarrollo empresarial y académico-investigativo del Quindío, a quienes se consultó mediante una entrevista semiestructurada, acerca del cuestionario y a la vez se buscó determinar posibles factores adicionales que influyen en la intención de crear empresas entre los estudiantes universitarios del Quindío.

Una vez hecho este primer ajuste al modelo de encuesta, se seleccionó un grupo de 51 estudiantes universitarios de último semestre de diversas universidades y carreras profesionales en metodología presencial ("estudiantes jueces"), para que respondieran la encuesta depurada, tal como lo sugieren Padua, et al., 
$(2013)^{5}$. La composición del grupo de "estudiantes jueces" se detalla en la Tabla 2 y se elaboró de manera proporcional al número de estudiantes de la población analizada, cuidando de excluir a dichos expertos de la encuesta final, que se aplicó posteriormente a la muestra seleccionada para obtener información primaria en un proyecto de investigación.

El trabajo de los estudiantes jueces pretendía identificar situaciones relacionadas con aspectos como los siguientes: la priorización de las preguntas más discriminantes, entendimiento y claridad de los ítemes formulados, ítemes adicionales que se podían incluir o replantear, tiempo de respuesta y la opción para que los encuestados pudieran manifestar su opinión de manera abierta sobre algunos factores que motivan y obstaculizan la creación de empresas (actitudes positivas y negativas).

Tabla 1. Grupos de preguntas, variables y autores de quienes se adaptaron las preguntas de la encuesta inicial

\begin{tabular}{|c|c|c|}
\hline Dimensión & Variables & Autores \\
\hline $\begin{array}{l}\text { Aspectos familiares } \\
\text { y personales }\end{array}$ & $\begin{array}{l}\text { - Estado civil } \\
\text { - Edad } \\
\text { - Género } \\
\text { - Campo de estudio } \\
\text { - Clase social } \\
\text { - Nivel de estudios de los padres } \\
\text { - Ocupación de los padres } \\
\text { - Familiares empresarios } \\
\text { - Experiencia laboral. } \\
\text { - Amigos empresarios cercanos. }\end{array}$ & $\begin{array}{l}\text { Gasse \& Tremblay (2011) } \\
\text { Tarapuez (2008) } \\
\text { Bhandari (2012) } \\
\text { Fayolle, et al., (2006) } \\
\text { Kantis, et al., (2014) } \\
\text { Solimano (2014) } \\
\text { Urbano (2006) } \\
\text { Scott \& Twomey (1988) } \\
\text { Birdthistle (2008) } \\
\text { Turker \& Sonmes (2009). }\end{array}$ \\
\hline Normas sociales & $\begin{array}{l}\text { - Empresa vs. poder, respeto y esta- } \\
\text { tus social. } \\
\text { - Admiración por los empresarios } \\
\text { - Empresarios vs. Empleado }\end{array}$ & $\begin{array}{l}\text { Hofstede }(2001,1991,1980) \\
\text { Autio et al }(1997) \\
\text { Begley et al., (1997) } \\
\text { Urbano (2006) } \\
\text { Solimano (2014) } \\
\text { Boissin, et al (2009) } \\
\text { GEM Colombia (2013) }\end{array}$ \\
\hline $\begin{array}{l}\text { Imagen y valores } \\
\text { del empresario } \\
\text { quindiano }\end{array}$ & $\begin{array}{l}\text { - Asociatividad. } \\
\text { - Optimismo y visión de largo plazo. } \\
\text { - Predisposición para asumir riesgos } \\
\text { moderados. } \\
\text { - Creatividad e innovación. } \\
\text { - Autonomía. } \\
\text { - Capacidad de organización. }\end{array}$ & $\begin{array}{l}\text { Hofstede }(2001,1991,1980) \\
\text { Gasse \& Tremblay }(2011) \\
\text { Autio, et al., (1997) } \\
\text { Solimano (2014) } \\
\text { Urbano (2006) } \\
\text { Obisanya, et al., (2010) } \\
\text { GEM Colombia (2013) } \\
\text { Entrevistas expertos locales }\end{array}$ \\
\hline
\end{tabular}

5. Padua, et al.,(2013:170) sugieren construir de 30 a 50 ítems y seleccionar una muestra de jueces entre 50 a 100 personas, dichos jueces deben ser seleccionados al azar de una población con características similares a aquella a la que se pretende aplicar la escala final. La metodología de Padua. et al., (2013) fue seguida en la definición de los factores que más discriminan las variable de estudio. 


\begin{tabular}{|c|c|c|}
\hline Dimensión & Variables & Autores \\
\hline $\begin{array}{l}\text { Creencias acerca } \\
\text { de la creación de } \\
\text { empresas }\end{array}$ & $\begin{array}{l}\text { - Importancia del dinero. } \\
\text { - Participación en cursos y progra- } \\
\text { mas de asesoría empresarial. } \\
\text { - Materias de emprendimiento en la } \\
\text { universidad. } \\
\text { - Papel de la familia. } \\
\text { - Dedicación de tiempo. }\end{array}$ & $\begin{array}{l}\text { Gasse \& Tremblay (2011) } \\
\text { Autio, et al.,(1997) } \\
\text { Birdthistle (2008) } \\
\text { Falloye, et al., (2006) } \\
\text { Boissin, et al.,(2009) }\end{array}$ \\
\hline $\begin{array}{l}\text { Motivaciones } \\
\text { (actitudes positivas) } \\
\text { y obstáculos para } \\
\text { crear empresa } \\
\text { (actitudes } \\
\text { negativas) }\end{array}$ & $\begin{array}{l}\text { - Autorrealización. } \\
\text { - Reto personal. } \\
\text { - Independencia. } \\
\text { - Plan de vida. } \\
\text { - Insuficiente apoyo. } \\
\text { - Falta de conocimiento en produc- } \\
\text { ción. } \\
\text { - Escaso capital. } \\
\text { - Creencias religiosas. } \\
\text { - Baja autoconfianza. } \\
\text { - Obligaciones académicas. } \\
\text { - Tipo de carrera universitaria. } \\
\text { - Falta claridad sobre qué empresa } \\
\text { - Frear. } \\
\text { - Falta de socios. }\end{array}$ & $\begin{array}{l}\text { Hofstede }(2001,1991,1980) \\
\text { Gasse \& Tremblay }(2011) \\
\text { Urbano (2006) } \\
\text { Solimano (2014) } \\
\text { Birdthistle (2008) } \\
\text { Obisanya, et al., (2010) }\end{array}$ \\
\hline $\begin{array}{l}\text { Recursos (locus de } \\
\text { control) }\end{array}$ & $\begin{array}{l}\text { - Sector económico de la empresa } \\
\text { por crear } \\
\text { - Plazo para crear la empresa } \\
\text { - Identificación oportunidades de } \\
\text { negocio } \\
\text { - Recursos para crear la empresa }\end{array}$ & $\begin{array}{l}\text { Tarapuez (2008) } \\
\text { Gasse \& Tremblay (2011) } \\
\text { Urbano (2006) } \\
\text { Birdthistle (2008) } \\
\text { Bhandari (2012) } \\
\text { Bakotic, et al., (2010) }\end{array}$ \\
\hline
\end{tabular}

Fuente: elaboración propia

Hubo acuerdo con el grupo de expertos, tanto estudiantes como profesionales, en que las preguntas priorizadas constituyen una base adecuada de interrogantes para determinar los aspectos que influyen en la intención de crear empresas, entre los estudiantes universitarios de último semestre.

La selección de las preguntas que discriminan, se hizo mediante una comparación de dos grupos: uno conformado por los puntajes menores o iguales al primer cuartil, identificado como "bajo" y el otro conformado por los puntajes mayores o iguales al tercer cuartil, identificado como "alto"; se consideraron preguntas que discriminan, aquellas en las cuales existe diferencia significativa entre los dos grupos; para este proceso se utilizó la prueba de Kruskall y Wallis en el programa Statgraphics Centurion; en la Tabla 3 se resumen los ítemes seleccionados. 
Tabla 2. Población y muestra de estudiantes universitarios de último semestre del departamento del Quindío (Colombia), 2014

\begin{tabular}{|c|l|c|c|c|c|}
\hline No. & \multicolumn{1}{|c|}{ Universidad } & Sigla & $\begin{array}{c}\text { Número de } \\
\text { estudiantes } \\
\text { (población) }\end{array}$ & $\begin{array}{c}\text { Número de } \\
\text { encuestas a } \\
\text { aplicar } \\
\text { (muestra) }\end{array}$ & $\begin{array}{c}\text { Número de } \\
\text { "estudiantes } \\
\text { jueces" }\end{array}$ \\
\hline 1 & Universidad del Quindío & UQ & 677 & 155 & 26 \\
\hline 2 & Fundación Universitaria San Martín & FUSM & 73 & 17 & 3 \\
\hline 3 & Universidad La Gran Colombia & UGC & 283 & 64 & 11 \\
\hline 4 & $\begin{array}{l}\text { Escuela de Administración y } \\
\text { Mercadotecnia }\end{array}$ & EAM & 84 & 19 & 3 \\
\hline 5 & $\begin{array}{l}\text { Universidad Alexander Von } \\
\text { Humboldt }\end{array}$ & UAVH & 127 & 29 & 5 \\
\hline 6 & Universidad Antonio Nariño & UAN & 23 & 5 & 1 \\
\hline 7 & Universidad San Buenaventura & USB & 37 & 8 & 2 \\
\hline---- & & ----- & 1.304 & 297 & 51 \\
\hline
\end{tabular}

* Corresponde a la muestra del trabajo de campo de la investigación que se realizaría posteriormente.

Fuente: elaboración propia con información de cada universidad.

Tabla 3. Priorización de las preguntas más discriminantes que se utilizaron en la encuesta final

\begin{tabular}{|c|c|c|c|c|}
\hline \multirow{3}{*}{$\begin{array}{c}\text { Grupo de variables } \\
\text { Pregunta }\end{array}$} & $\begin{array}{c}\text { Código } \\
\text { encuesta } \\
\text { inicial }\end{array}$ & $\begin{array}{c}\text { Ítem de la } \\
\text { Prueba de Kruskal-Wallis } \\
\text { (p-valor) }\end{array}$ & ¿Discrimina? \\
\hline \multirow{3}{*}{$\begin{array}{c}\text { Normas sociales } \\
\text { (3 preguntas) }\end{array}$} & 1 & 1.1 & 0,026 & Sí \\
\cline { 2 - 5 } & 4 & $1.4^{*}$ & & No \\
\cline { 2 - 5 } & 6 & 1.6 & 0,0275 & Sí \\
\hline \multirow{4}{*}{$\begin{array}{c}\text { Imagen y valores } \\
\text { (6 preguntas) }\end{array}$} & 12 & 2.1 & 0,0092 & Sí \\
\cline { 2 - 5 } & 13 & 2.2 & 0,0004 & Sí \\
\cline { 2 - 5 } & 16 & 2.8 & 0,0199 & Sí \\
\cline { 2 - 5 } & 17 & 2.9 & 0,0204 & Sí \\
\hline
\end{tabular}




\begin{tabular}{|c|c|c|c|c|}
\hline \multirow{6}{*}{$\begin{array}{c}\text { Creencias } \\
\text { (6 preguntas) }\end{array}$} & 21 & 3.1 & 0,0887 & Sí \\
\hline & 23 & $3.3^{* *}$ & & No \\
\hline & 24 & 3.4 & 0,0419 & Sí \\
\hline & 25 & 3.5 & 0,0012 & Sí \\
\hline & 29 & 3.9 & 0,0232 & Sí \\
\hline & 31 & 3.11 & 0,0601 & Sí \\
\hline \multirow{4}{*}{$\begin{array}{l}\text { Aspectos que } \\
\text { motivan } \\
\text { (4 preguntas) }\end{array}$} & 32 & 4.1 & 0,0076 & Sí \\
\hline & 34 & 4.3 & 0,016 & Sí \\
\hline & 35 & 4.4 & 0,0045 & Sí \\
\hline & 38 & 4.7 & 0,0014 & Sí \\
\hline \multirow{9}{*}{$\begin{array}{c}\text { Obstáculos para crear } \\
\text { empresa } \\
\text { (9 preguntas) }\end{array}$} & 40 & 5.1 & 0,0024 & Sí \\
\hline & 41 & 5.2 & 0,0045 & Sí \\
\hline & 42 & 5.3 & 0,001 & Sí \\
\hline & 44 & $5.5^{* *}$ & & No \\
\hline & 45 & 5.6 & 0,0035 & Sí \\
\hline & 46 & 5.7 & 0,00148 & Sí \\
\hline & 47 & 5.8 & 0,00007 & Sí \\
\hline & 49 & 5.10 & 0,00056 & Sí \\
\hline & 50 & 5.11 & 0,00023 & Sí \\
\hline
\end{tabular}

* A pesar de que esta pregunta no discrimina lo necesario, se incluye por recomendación de uno de los evaluadores del anteproyecto de tesis doctoral, quien sugirió analizar las dimensiones de Hofstede; el poder es una de dichas dimensiones.

** Teniendo en cuenta el problema analizado en la investigación, en la reunión de expertos se decidió incluir esta variable dada la naturaleza de la población que se estudia.

La discusión del modelo final de encuesta se hizo con un subgrupo de los 14 líderes regionales, en la que se socializaron y discutieron las preguntas priorizadas. Como fruto de esta actividad y considerando las particularidades sociales e históricas de la región, se decidió incluir adicionalmente las preguntas No. 23 y 44, correspondientes a los ítems 3.3 y 5.5, y la pregunta 4 correspondiente al ítem 1.4 por recomendación de uno de los evaluadores del proyecto de investigación (Tabla 3, véase las notas al pie de la misma).

\section{b) Prueba piloto}

Con el propósito de validar la encuesta se procedió a realizar una prueba piloto con una muestra de 30 estudiantes, distribuidos proporcionalmente en las diferentes universidades. Las encuestas se aplicaron y tabularon de la siguiente manera: la mitad en formato físico y la mitad utilizando la página de internet www. encuestafacil.com. La página de internet generó automáticamente una matriz de 
datos en Excel que fue complementada manualmente con la información de las encuestas en papel.

Los resultados de la aplicación en la prueba piloto mostraron que era necesario adecuar la encuesta electrónica para evitar que los estudiantes que no tenían intención empresarial pudieran dar por terminada su encuesta en la pregunta No. 30, ya que de ahí en adelante ellos no debían responder más interrogantes.

\section{COMPRENSIÓN DE LA ENCUESTA}

Durante la priorización de preguntas y la prueba piloto, se comprobó que el modelo de encuesta contaba con una adecuada distribución que permitía una buena sucesión de los temas, con buena redacción y de fácil entendimiento.

\section{MODELO DEFINITIVO DE LA ENCUESTA}

Finalmente se trabajó con las 28 preguntas tipo Likert, adicionalmente a 14 preguntas relacionadas con aspectos personales y familiares, y 4 preguntas sobre recursos $^{6}$. Se hicieron algunos cambios en el modelo de encuesta para que los estudiantes de la muestra pudieran identificar claramente qué preguntas tenían escala positiva y cuáles escala negativa. Se hicieron las adecuaciones del caso para que el cuestionario se acomodara a un máximo de 5 páginas. En algunas preguntas se incluyó información más detallada buscando mayor claridad y respuestas de fácil identificación.

\section{UTILIDAD DEL CONSTRUCTO}

La aplicación de la encuesta permitió obtener información primaria, para el proyecto: "Factores que influyen en la intención de creación de empresas en los estudiantes universitarios del departamento del Quindío (Colombia)”, que fue procesada utilizando las técnicas: Correspondencias Múltiples, Pruebas de Kruskal y Wallis, Regresión Logística y Análisis Factorial Confirmatorio; los resultados obtenidos de los análisis se ajustan a la Teoría del Comportamiento Planeado aunque se identificaron influencias adicionales directas e indirectas sobre la intención empresarial.

\section{RESULTADOS}

El resultado final se concreta en la encuesta que aparece como anexo y que también se puede consultar en: https://www.researchgate.net/profile/Edwin_Tarapuez/publications?pubType=technicalReport

\section{CONCLUSIONES}

El proceso utilizado en la construcción de una encuesta orientada a identificar los principales factores para medir la intención empresarial en estudiantes

6. Las preguntas sobre aspectos personales y familiares, y sobre recursos, fueron concertadas en las reuniones con los expertos regionales. 
universitarios de último semestre, agrupando las variables de estudio de acuerdo con la Teoría del Comportamiento Planeado, agregando otros grupos de variables relacionadas con aspectos personales y familiares, factores socioculturales, creencias y valores, y finalmente priorizando las preguntas, se concretó en el diseño de un instrumento para recoger la información primaria en el proyecto "Factores que influyen en la intención de creación de empresas en los estudiantes universitarios del departamento del Quindío (Colombia)”.

La elaboración de un instrumento para obtener la información primaria en proyectos de investigación social, es una tarea que, además de la consulta bibliográfica y el análisis de situaciones similares en otros contextos, requiere el aporte de expertos conocedores del medio donde se aplicará y de personas de la población objeto.

\section{REFERENCIAS}

1. AJZEN, I. (1991). The theory of planned behavior. Organitational Behavior and Human Decision Proceses. 50, 179-211.

2. AJZEN, I. (1988). Attitudes, Personality and Behavior. Chicago, The Dorsey Press.

3. AJZEN, I. (1987): Attitudes, traits and Actions: dispositional predictions of behavior in Personality and Social Psychology. Berkowitz, L. (ed.). Advances in Experimental Social Psychology. Nueva York, Academic. 20, 1-63.

4. AJZEN, I. (1985). From intentions to actions: A Theory of Planned Behavior. Khul, J. \& Beckmann, J. (Eds). Action control from cognition to behavior. Germany, Springer-Verlag. 31. 10-39.

5. BAUM, J., FRESE, M., BARON, R. \& KATZ, J. (2007). Entrepreneurship as an Area of Psychology Study: An Introduction. Baum, J., Frese, M. \& Baron, R. (Eds). The Psychology of Entrepreneurship. New Jersey, Society for Industrial and Organizational Psychology. 1-18.

6. BIRD, B. (1988). Implementing entrepreneurial ideas: the case for intention. Academy of Management Review. 13 (3). 442-453.

7. CONGRESO DE COLOMBIA (2012). Ley 1581. Régimen General de Protección de Datos Personales.

8. GASSE, Y. \& D’AMOURS, A. (2000). Profession: entrepreneur. Montreal, Transcontinental.

9. GASSE, Y. \& TREMBLAY, M. (2011). Entrepreneurial Beliefs and Intentions: A Cross-Cultural Study of University Students in Seven Countries. International Journal Of Business. 16(4). 1083-4346. 
10. GATEWOOD, E., SHAVER, K. \& GARTNER, W. (1995). A longitudinal study of cognitive factors influencing start-up behaviors and success at venture creation. Journal of Business Venturing. 10 (5). 371-91.

11. GEM Colombia (2013). Dinámica Empresarial Colombiana. Bogotá, D.C. Universidad Icesi, Universidad del Norte, Universidad de los Andes, Pontificia Universidad Javeriana Cali.

12. HARRIS, M. \& GIBSON, S. (2008). Examining the entrepreneurial attitudes of US business students. Education + Training. 50 (7). 568-581.

13. HISRICH, R., PETERS, M. \& SHEPHERD, D. (2004), Entrepreneurship. New York, Irwin-McGraw Hill. 6th ed.

14. HOFSTEDE, G. (1980). Motivation, leadership, and organizations: do American theories apply abroad? Organizational Dynamics, 9 (1). 42-63.

15. HOFSTEDE, G. (1991). Culture and organizations. Londres, McGraw-Hill.

16. HOFSTEDE, G. (2001). Culture's consequences: Comparing values, behaviors, institutions, and organizations across nations. California, Sage Publications.

17. JONES, P., JONES, A., PACKHAM, G. \& MILLER, C. (2008). Student attitudes towards enterprise education in Poland: a positive impact. Education + Training. 50 (7). 597-614.

18. KRUEGER, N. \& BRAZEAL, D. (1994). Entrepreneurial Potential and Potential Entrepreneurs. Entrepreneurship Theory and Practice. 18 (3). 91-104.

19. KRUEGER, N. (1993). The Impact of Prior Entrepreneurial Exposure on Perceptions of New Venture Feasibility and Desirability. Entrepreneurship Theory and Practice. 18 (1). 5-21.

20. KRUEGER, N. \& CARSRUD. A. (1993). Entrepreneurial intentions: Applying the theory of planned behavior. Entrepreneurship \& Regional Development: An International Journal. 5 (4). 315-330.

21. KURATKO, D. \& HODGETTS, R. (2006). Entrepreneurship. Florence, KY, South-Western College Publishing. 7th ed.

22. LIÑÁN, F. (2008). Skill and value perceptions: how do they affect entrepreneurial intentions? International Entrepreneurship and Management Journal. 4 (3). 257-272.

23. NABI, G. \& HOLDEN, R. (2008). Graduate entrepreneurship: intentions, education and training. Education + Training. 50 (7). 545-551.

24. SHAPERO, A. (1984). The entrepreneurial event. Kent, C. (Ed.). The environment for entrepreneurship. Lexington: Lexington Books.

25. TARAPUEZ, E. (2008). La creación de empresas en los estudiantes de Contaduría Pública de la Universidad del Quindío. Ponencia presentada en el IX Congreso de Contaduría Pública y III Congreso Internacional de Estudiantes de Contaduría Pública. Medellín, agosto 14 al 16. 


\section{Anexo \\ Universidad Nacional de Córdoba (Argentina) Doctorado en Ciencias Económicas}

Tesis doctoral: Factores que influyen en la intención de crear empresa en estudiantes universitarios de último semestre de carreras presenciales profesionales en el Departamento del Quindío (Colombia).

\section{MODELO DE INSTRUMENTO DE RECOLECCIÓN DE INFORMACIÓN PRIMARIA}

Autor: Edwin Tarapuez Chamorro. Email: eitarapuez@uniquindio.edu.co \begin{tabular}{|l|l}
\hline Fecha: ___ _ _ 2014 & Encuestador (a):
\end{tabular}

Objetivo: determinar los factores que influyen en la intención de crear empresa en los estudiantes universitarios de último semestre del Quindío, carreras profesionales presenciales, 2014.

Dirigida a: Estudiantes de último semestre de carreras presenciales profesionales de las universidades del departamento del Quindío.

Para esta encuesta es importante que usted tenga en cuenta dos conceptos particulares:

Emprendedor: es la persona que realiza algún tipo de actividad productiva y/o comercial, enfocada a desarrollar sus habilidades personales y hacia la generación de ingresos, ya sea que la realice de manera formal o informal, independientemente del tiempo que se dedique a ella.

Empresa o negocio: cualquier actividad legal, productiva, comercial o de servicios, de propiedad del estudiante, total o parcialmente, que le genera ingresos adicionales.

Nombre y apellido:

Email: Tel. o celular:

1. Estado civil: OC

1. Casado $\square$ 2. Soltero $\square \quad$ 3. Separado $\square \quad$ 4. Viudo

5. Unión libre $\square \quad$ 6. Divorciado $\square \quad$ 7. No sabe, no responde

2. Edad: años OA

3. Género (marque con X. Respuesta única): ОА

\begin{tabular}{|l|l|l|}
\hline 1 & Masculino & \\
\hline
\end{tabular}

\begin{tabular}{|l|l|l|}
\hline 2 & Femenino & \\
\hline
\end{tabular}


4. ¿A qué estrato socioeconómico pertenece? (marque con X. Respuesta única): OA-C

\begin{tabular}{|c|c|c|c|c|c|c|}
\hline 1 & 2 & 3 & 4 & 5 & 6 & No sabe/No responde \\
\hline
\end{tabular}

5. ¿En qué universidad (es) estudia? OA-C
1. U. Quindío
2. U. San Martín
3. La Gran Colombia

4. EAM

5. U. Antonio Nariño

6. U. Alexander Von Humboldt $\square$

7. U. San Buenaventura

6. ¿Cuál es el área de la carrera universitaria que estudia? (Marque una sola opción)

$\square$ CIENCIAS ECONÓMICAS Y ADMINISTRATIVAS (Economía, Administración, Contaduría pública, Finanzas, Relaciones Internacionales, Publicidad y mercadeo).

$\square$ CIENCIAS DE LA SALUD (Medicina, Psicología, Salud ocupacional, Odontología, Enfermería).

$\square$ INGENIERÍA Y ARQUITECTURA (Ingeniería de sistemas y computación, Ingeniería civil, Ingeniería electrónica, Ingeniería agroindustrial, Ingeniería industrial, Ingeniería de alimentos, Ingeniería mecatrónica, Arquitectura).

$\square$ CIENCIAS SOCIALES y HUMANAS (Derecho, Ciencias de la información, Comunicación social y periodismo, Gerontología, Trabajo social).

$\square$ CIENCIAS BÁSICAS O LICENCIATURAS EN EDUCACIÓN (Biología, Física, Química, Lenguas modernas, Biología y Educación ambiental, Español y literatura, Educación física, Matemáticas, Filosofía, Pedagogía, Ciencias sociales, Bellas Artes, Música).

$\square$ CIENCIAS AGROPECUARIAS (Medicina veterinaria, Agronomía, Zootecnia)

$\square$ Otra (Por favor especifique)

7-8. ¿Cuál es o fue el NIVEL MÁXIMO de estudios formales alcanzado por su padre y su madre? (marque con X para cada uno de ellos. Respuesta única): oc

\begin{tabular}{|c|c|c|c|c|c|}
\hline No. & Nivel de estudios & Padre & \multirow{7}{*}{$\begin{array}{l}\text { No sabe, } \\
\text { no } \\
\text { responde } \\
\square\end{array}$} & Madre & \multirow{7}{*}{$\begin{array}{c}\text { No sabe, } \\
\text { no } \\
\text { responde }\end{array}$} \\
\hline 1 & No asistió al colegio & & & & \\
\hline 2 & Primaria o primaria incompleta & & & & \\
\hline 3 & Secundaria o secundaria incompleta & & & & \\
\hline 4 & Técnica o tecnológica, completa o incompleta & & & & \\
\hline 5 & Universitaria o universitaria incompleta & & & & \\
\hline 6 & Postgrado o postgrado incompleto & & & & \\
\hline
\end{tabular}


9-10. ¿Cuál ha sido la PRINCIPAL ocupación de su padre y de su madre? (marque con X para cada uno de ellos. Respuesta única, marque con X): OC

\begin{tabular}{|c|c|c|c|c|c|}
\hline No. & Ocupación & Padre & \multirow{6}{*}{$\begin{array}{l}\text { No } \\
\text { sabe, no } \\
\text { responde } \\
\square\end{array}$} & Madre & \multirow{6}{*}{$\begin{array}{l}\text { No } \\
\text { sabe, no } \\
\text { responde }\end{array}$} \\
\hline 1 & Empleado o contratista en empresa privada o pública & & & & \\
\hline 2 & Comerciante, emprendedor, empresario. & & & & \\
\hline 3 & Trabajador independiente (por su propia cuenta) & & & & \\
\hline 4 & Labores domésticas en su propio hogar & & & & \\
\hline 5 & Otra ¿Cuál? & & & & \\
\hline
\end{tabular}

11. Responda las siguientes preguntas (Respuesta única, marque con $\mathrm{X}$ ):

\begin{tabular}{|c|l|l|l|l|}
\hline No. & \multicolumn{1}{|c|}{ Pregunta } & Si & No & $\begin{array}{c}\text { No sabe / } \\
\text { No responde }\end{array}$ \\
\hline 11.1 & $\begin{array}{l}\text { ¿Alguno de los miembros de su FAMILIA (padres, } \\
\text { hermanos o abuelos) tiene un negocio o empresa } \\
\text { propia? oA }\end{array}$ & & \\
\hline 11.2 & $\begin{array}{l}\text { ¿Tiene algún AMIGO muy cercano que sea dueño de } \\
\text { su propia empresa que haya influido en su visión de } \\
\text { los negocios? oA }\end{array}$ & & \\
\hline 11.3 & $\begin{array}{l}\text { ¿Tiene usted alguna experiencia laboral o ha sido } \\
\text { contratado por una empresa para desarrollar algún } \\
\text { trabajo? oc }\end{array}$ & & \\
\hline
\end{tabular}

12. Por favor indique en qué medida está de acuerdo o en desacuerdo con cada declaración sobre las NORMAS SOCIALES que influyen en la intención de crear empresa (marque con $\mathrm{X}$ una sola respuesta por cada fila): $\mathrm{OB}$

\begin{tabular}{|l|l|c|c|c|c|c|}
\hline No. & \multicolumn{1}{|c|}{ Ítems } & $\begin{array}{c}\text { Totalmente } \\
\text { de acuerdo }\end{array}$ & $\begin{array}{c}\text { De } \\
\text { acuerdo }\end{array}$ & Neutro & $\begin{array}{c}\text { En } \\
\text { desacuerdo }\end{array}$ & $\begin{array}{c}\text { Totalmente } \\
\text { en } \\
\text { desacuerdo }\end{array}$ \\
\hline 12.1 & $\begin{array}{l}\text { Es mejor ser dueño de } \\
\text { su propia empresa que } \\
\text { empleado en una ajena. }\end{array}$ & (5) $\square$ & (4) $\square$ & (3) $\square$ & (2) $\square$ & (1) $\square$ \\
\hline 12.2 & $\begin{array}{l}\text { En esta región se admira } \\
\text { a quienes dirigen sus } \\
\text { propias empresas }\end{array}$ & (5) $\square$ & (4) $\square$ & (3) $\square$ & (2) $\square$ & (1) $\square$ \\
\hline 12.3 & $\begin{array}{l}\text { Tener una empresa propia } \\
\text { genera poder, respeto y } \\
\text { un estatus social alto. } \mathrm{HF}\end{array}$ & (5) $\square$ & (4) $\square$ & (3) $\square$ & (2) $\square$ & (1) $\square$ \\
\hline
\end{tabular}


13. Valore cada uno de los siguientes atributos relativos a IMAGEN y VALORES sobre los EMPRESARIOS QUINDIANOS (marque con X una sola respuesta por cada fila): oD-G

\begin{tabular}{|l|l|c|c|c|c|c|}
\hline No. & \multicolumn{1}{|c|}{ Ítems } & $\begin{array}{c}\text { Totalmente } \\
\text { de acuerdo }\end{array}$ & $\begin{array}{c}\text { De } \\
\text { acuerdo }\end{array}$ & Neutro & $\begin{array}{c}\text { En } \\
\text { desacuerdo }\end{array}$ & $\begin{array}{c}\text { Totalmente } \\
\text { en } \\
\text { desacuerdo }\end{array}$ \\
\hline 13.1 & $\begin{array}{l}\text { Les gusta asociarse con otros } \\
\text { empresarios e inversionistas. HF }\end{array}$ & (5) $\square$ & (4) $\square$ & (3) $\square$ & (2) $\square$ & (1) $\square$ \\
\hline 13.2 & $\begin{array}{l}\text { Demuestran su mentalidad } \\
\text { freativa e innovadora. }\end{array}$ & (5) $\square$ & (4) $\square$ & (3) $\square$ & (2) $\square$ & (1) $\square$ \\
\hline 13.3 & $\begin{array}{l}\text { Asumen riesgos calculados para } \\
\text { el crecimiento de sus empresas } \\
\text { HF }\end{array}$ & (5) $\square$ & (4) $\square$ & (3) $\square$ & (2) $\square$ & (1) $\square$ \\
\hline 13.4 & $\begin{array}{l}\text { Son optimistas y tienen visión } \\
\text { de largo plazo. HF }\end{array}$ & (5) $\square$ & (4) $\square$ & (3) $\square$ & (2) $\square$ & (1) $\square$ \\
\hline 13.5 & $\begin{array}{l}\text { Son autónomos para desarrollar } \\
\text { sus iniciativas. }\end{array}$ & (5) $\square$ & (4) $\square$ & (3) $\square$ & (2) $\square$ & (1) $\square$ \\
\hline 13.6 & $\begin{array}{l}\text { Tienen gran capacidad de } \\
\text { organización. }\end{array}$ & (5) $\square$ & (4) $\square$ & (3) $\square$ & (2) $\square$ & (1) $\square$ \\
\hline
\end{tabular}

14. Valore cada una de las siguientes CREENCIAS sobre la creación de empresas (marque con $\mathrm{X}$ una sola respuesta por cada fila):OD

\begin{tabular}{|l|l|r|r|r|r|r|}
\hline No & \multicolumn{1}{|c|}{ Ítems } & $\begin{array}{l}\text { Totalmente } \\
\text { de acuerdo }\end{array}$ & $\begin{array}{c}\text { De } \\
\text { acuerdo }\end{array}$ & Neutro & $\begin{array}{c}\text { En } \\
\text { desacuerdo }\end{array}$ & $\begin{array}{l}\text { Totalmente } \\
\text { en } \\
\text { desacuerdo }\end{array}$ \\
\hline 14.1 & $\begin{array}{l}\text { La familia desempeña un } \\
\text { papel positivo en la creación y } \\
\text { desarrollo de la empresa. }\end{array}$ & (5) & (4) & (3) $\square$ & (2) $\square$ & (1) \\
\hline 14.2 & $\begin{array}{l}\text { Participar en cursos y } \\
\text { programas de asesoría } \\
\text { empresarial aumenta la } \\
\text { intención de crear una empresa }\end{array}$ & (5) $\square$ & (4) $\square$ & (3) $\square$ & (2) $\square$ & (1) \\
\hline 14.3 & $\begin{array}{l}\text { El dinero es solamente un factor } \\
\text { más de los que se requiere para } \\
\text { crear empresa. }\end{array}$ & (5) $\square$ & (4) $\square$ & (3) $\square$ & (2) $\square$ & (1) \\
\hline 14.4 & $\begin{array}{l}\text { Las materias de } \\
\text { emprendimiento de la } \\
\text { universidad desarrollan mi } \\
\text { intención de crear una empresa. }\end{array}$ & (5) $\square$ & (4) $\square$ & (3) $\square$ & (2) $\square$ & (1) $\square$ \\
\hline 14.5 & $\begin{array}{l}\text { Ser empresario requiere más } \\
\text { dedicación que si se decide ser } \\
\text { empleado en una empresa }\end{array}$ & (5) $\square$ & (4) $\square$ & (3) $\square$ & (2) $\square$ & (1) $\square$ \\
\hline 14.6 & $\begin{array}{l}\text { Ganar dinero es lo único que le } \\
\text { interesa a los empresarios }\end{array}$ & (5) $\square$ & (4) $\square$ & (3) $\square$ & (2) $\square$ & (1) $\square$ \\
\hline
\end{tabular}


15. ¿Alguna vez ha tenido usted la INTENCIÓN de crear una empresa o negocio propio? (marque con $\mathrm{X}$, respuesta única): $\mathrm{OF}$

\begin{tabular}{|c|l|c|}
\hline No & \multicolumn{1}{|c|}{ Ítem } & X \\
\hline 1 & Si, vagamente & \\
\hline 2 & No, nunca & \\
\hline 3 & No, pero pienso vincularme con una empresa de la familia & \\
\hline 4 & Si, seriamente & \\
\hline 5 & Tengo una empresa o negocio o actividad empresarial propia & \\
\hline
\end{tabular}

\section{SI SU RESPUESTA A LA PREGUNTA ANTERIOR ES "NO, NUNCA" POR FAVOR TERMINE AQUÍ SU ENCUESTA. GRACIAS}

16. Valore los siguientes factores que lo motivan, motivaron o motivarían en su INTENCIÓN para crear una empresa propia, de acuerdo con la escala propuesta: $\mathrm{OF}$

\begin{tabular}{|l|l|c|c|c|c|c|}
\hline No & \multicolumn{1}{|c|}{ Motivaciones para crear empresa } & $\begin{array}{c}\text { Totalmente } \\
\text { de acuerdo }\end{array}$ & $\begin{array}{c}\text { De } \\
\text { acuerdo }\end{array}$ & Neutro & $\begin{array}{c}\text { En } \\
\text { desacuerdo }\end{array}$ & $\begin{array}{c}\text { Totalmente } \\
\text { en } \\
\text { desacuerdo }\end{array}$ \\
\hline 16.1 & $\begin{array}{l}\text { Identificar y superar un reto } \\
\text { personal }\end{array}$ & (5) $\square$ & (4) $\square$ & (3) $\square$ & (2) $\square$ & (1) $\square$ \\
\hline 16.2 & $\begin{array}{l}\text { Ser mi propio jefe, manejar } \\
\text { mi tiempo y alcanzar la } \\
\text { independencia }\end{array}$ & (5) $\square$ & (4) $\square$ & (3) $\square$ & (2) $\square$ & (1) $\square$ \\
\hline 16.3 & $\begin{array}{l}\text { Lograr mi autorrealización y } \\
\text { satisfacción personal } \mathrm{HF}\end{array}$ & (5) $\square$ & (4) $\square$ & (3) $\square$ & (2) $\square$ & (1) $\square$ \\
\hline 16.4 & $\begin{array}{l}\text { En su plan de vida no se visualiza } \\
\text { como empleado en una empresa } \\
\text { ya creada }\end{array}$ & (5) $\square$ & (4) $\square$ & (3) $\square$ & (2) $\square$ & (1) $\square$ \\
\hline
\end{tabular}

17. Valore los siguientes factores que lo desmotivan, desmotivaron o desmotivarían en su INTENCIÓN para crear una empresa propia, de acuerdo con la escala propuesta: $\mathrm{OF}$

\begin{tabular}{|l|l|c|c|c|c|c|}
\hline No & \multicolumn{1}{|c|}{ Obstáculos para crear empresa } & $\begin{array}{c}\text { Totalmente } \\
\text { de acuerdo }\end{array}$ & $\begin{array}{c}\text { De } \\
\text { acuerdo }\end{array}$ & Neutro & $\begin{array}{c}\text { En } \\
\text { desacuerdo }\end{array}$ & $\begin{array}{c}\text { Totalmente } \\
\text { en } \\
\text { desacuerdo }\end{array}$ \\
\hline 17.1 & $\begin{array}{l}\text { Escaso capital para iniciar la } \\
\text { empresa }\end{array}$ & (1) $\square$ & (2) $\square$ & (3) $\square$ & (4) $\square$ & (5) $\square$ \\
\hline 17.2 & $\begin{array}{l}\text { Demasiadas obligaciones } \\
\text { académicas en la universidad }\end{array}$ & (1) $\square$ & (2) $\square$ & (3) $\square$ & (4) $\square$ & (5) $\square$ \\
\hline 17.3 & $\begin{array}{l}\text { Insuficiente apoyo de entidades } \\
\text { públicas y privadas para crear la } \\
\text { empresa. }\end{array}$ & (1) $\square$ & (2) $\square$ & (3) $\square$ & (4) $\square$ & (5) $\square$ \\
\hline
\end{tabular}




\begin{tabular}{|l|l|c|c|c|c|c|}
\hline 17.4 & $\begin{array}{l}\text { Mi carrera universitaria no es para } \\
\text { crear empresa. }\end{array}$ & (1) $\square$ & (2) $\square$ & (3) & (4) $\square$ & (5) $\square$ \\
\hline 17.5 & $\begin{array}{l}\text { Algunas creencias religiosas } \\
\text { castigan a los ricos y promueven la } \\
\text { pobreza. }\end{array}$ & (1) $\square$ & (2) $\square$ & (3) $\square$ & (4) $\square$ & (5) $\square$ \\
\hline 17.6 & $\begin{array}{l}\text { Baja confianza en sus capacidades } \\
\text { emprendedoras }\end{array}$ & (1) $\square$ & (2) $\square$ & (3) $\square$ & (4) $\square$ & (5) $\square$ \\
\hline 17.7 & $\begin{array}{l}\text { No tiene muy claro qué empresa } \\
\text { va a crear }\end{array}$ & (1) $\square$ & (2) $\square$ & (3) $\square$ & (4) $\square$ & (5) $\square$ \\
\hline 17.8 & $\begin{array}{l}\text { Falta de socios para iniciar una } \\
\text { empresa }\end{array}$ & (1) $\square$ & (2) $\square$ & (3) $\square$ & (4) $\square$ & (5) $\square$ \\
\hline 17.9 & $\begin{array}{l}\text { No sé producir ni fabricar nada en } \\
\text { particular. }\end{array}$ & (1) $\square$ & (2) $\square$ & (3) $\square$ & (4) $\square$ & (5) $\square$ \\
\hline
\end{tabular}

18. ¿Cuándo estima que podría crear su empresa? (Marque con X. Respuesta única): OE-F

\begin{tabular}{|l|l|l|}
\hline 1 & Tan pronto termine sus estudios universitarios & \\
\hline 2 & Mucho tiempo después de terminar sus estudios & \\
\hline 3 & No sabe cuándo creará su negocio/empresa & \\
\hline 4 & Ya tengo mi propio negocio/empresa & \\
\hline
\end{tabular}

19. ¿A qué sector de la economía pertenece su empresa o la idea de negocio que posee o que tiene intención de crear? (Marque con X. Respuesta única): OA-E

\begin{tabular}{|l|l|l|}
\hline 1 & Industrial y agroindustrial & \\
\hline 2 & Comercial y servicios & \\
\hline 3 & Agropecuario & \\
\hline 4 & Tecnología & \\
\hline 5 & Otro. ¿Cuál? & \\
\hline
\end{tabular}

20. ¿Cómo identifica o cree que puede identificar las oportunidades o posibles opciones de creación de empresa? (Marque con una X. Respuesta única): oE

\begin{tabular}{|c|l|c|}
\hline 1 & Estudios previos que definen la vocación productiva de la región & \\
\hline 2 & $\begin{array}{l}\text { Proyectos y planes de negocio desarrollados por otros estudiantes en sus } \\
\text { trabajos de grado }\end{array}$ & \\
\hline 3 & $\begin{array}{l}\text { Mirando los negocios y sectores más dinámicos de la región, los que más } \\
\text { se mueven }\end{array}$ & \\
\hline 4 & Recomendaciones de sus familiares empresarios o amigos empresarios & \\
\hline 5 & Otro ¿Cuál? & \\
\hline
\end{tabular}


21. ¿Cuál cree que es el recurso MÁS IMPORTANTE que tiene si usted decidiera crear su empresa o si ya la ha creado? (Marque con una X. Respuesta única): oE

$\mathbf{X}$

\begin{tabular}{|c|l|c|}
\hline 1 & Una habilidad suya (saber producir algo, capacidad para vender, liderar) & \\
\hline 2 & Un plan de negocios o proyecto de empresa escrito & \\
\hline 3 & Su capacidad emprendedora de sacar las cosas adelante & \\
\hline 4 & Sus recursos económicos o los de su familia (dinero, ahorros, propiedades) & \\
\hline 5 & Una buena idea de negocio & \\
\hline 6 & Otro ¿Cuál? & \\
\hline
\end{tabular}

Observaciones y sugerencias: 